\title{
"Scar-cinoma": viewing the fibrotic lung mesenchymal cell in the context of cancer biology
}

\author{
Jeffrey C. Horowitz ${ }^{1}$, John J. Osterholzer ${ }^{1}$, Antonia Marazioti ${ }^{2}$ and \\ Georgios T. Stathopoulos ${ }^{2,3}$
}

Affiliations: ${ }^{1}$ Division of Pulmonary and Critical Care Medicine, Dept of Internal Medicine, University of Michigan Medical Center, Ann Arbor, MI, USA. ${ }^{2}$ Laboratory for Molecular Respiratory Carcinogenesis, Dept of Physiology, Faculty of Medicine, University of Patras, Rio, Greece. ${ }^{3}$ Comprehensive Pneumology Center and Institute for Lung Biology and Disease, University Hospital, Ludwig-Maximilians University and Helmholtz Zentrum München, Member of the German Center for Lung Research (DZL), Munich, Germany.

Correspondence: Jeffrey C. Horowitz, 6303 MSRB III, 1150 W Medical Center Dr, Ann Arbor, MI 48109-5642, USA. E-mail: jchorowaumich.edu

ABSTRACT Lung cancer and pulmonary fibrosis are common, yet distinct, pathological processes that represent urgent unmet medical needs. Striking clinical and mechanistic parallels exist between these distinct disease entities. The goal of this article is to examine lung fibrosis from the perspective of cancer-associated phenotypic hallmarks, to discuss areas of mechanistic overlap and distinction, and to highlight profibrotic mechanisms that contribute to carcinogenesis. Ultimately, we speculate that such comparisons might identify opportunities to leverage our current understanding of the pathobiology of each disease process in order to advance novel therapeutic approaches for both. We anticipate that such "outside the box" concepts could be translated to a more precise and individualised approach to fibrotic diseases of the lung.

@ERSpublications

Lung fibrosis and cancer are distinct, but share a number of cell signalling and phenotypic features http://ow.ly/Za9YB

Support statement: The authors' research is supported by US National Institutes of Health/National Heart, Lung and Blood Institute grant HL105489, and a Martin E. Galvin Fund for Pulmonary Fibrosis Research Pilot Grant (to J.C. Horowitz); a Merit Review Award from the Biomedical Laboratory Research and Development Service, US Dept of Veterans Affairs (to J.J. Osterholzer); European Research Council 2010 Starting Independent Investigator and 2015 Proof of Concept grants (260524 and 679345, respectively, to G.T. Stathopoulos); and European Respiratory Society, Hellenic Thoracic Society and Hellenic Association for Molecular Cancer Research scholarships and awards (to A. Marazioti and G.T. Stathopoulos). Funding information for this article has been deposited with FundRef

Conflict of interest: None declared.

Copyright OERS 2016 


\section{Introduction}

Idiopathic pulmonary fibrosis (IPF) is an interstitial lung disorder that is characterised, in part, by the excessive deposition and remodelling of extracellular matrix along with the accumulation and persistent activation of fibroblasts in the context of injury to the alveolar epithelium [1]. The 5-year survival of patients with IPF is comparable to that of lung cancer, which is among the most prevalent malignancies and is the leading cause of cancer-related mortality in the USA [2]. The incidence of IPF is increasing globally [3]. Yet, pharmacologic therapies have only recently been shown to limit progression of disease and have not been definitively shown to alter mortality, demonstrating an ongoing need for novel approaches to treatment $[4,5]$.

Clinically, patients with IPF and other fibrotic lung diseases have increased rates of lung cancer, with a reported prevalence as high as $48 \%$ in autopsy series of patients with usual interstitial pneumonia, the pathologic correlate of IPF [6-9]. Anatomically, the distribution of lung cancers in patients with IPF is predominantly lower lobe and peripheral, the same regions in which fibrosis is accentuated in IPF [8]. These associations have generated increasing interest in the clinical, anatomical and biological overlap between cancer and fibrosis [2, 7-16].

At the mechanistic level, lung fibrosis is considered to be the result of a dysregulated wound-repair response to lung injury characterised by the accumulation and persistence of activated reparative mesenchymal cells (myofibroblasts), which are responsible for the deposition of excessive extracellular matrix (ECM) [1]. Aberrant interactions between epithelial cells, mesenchymal cells and the ECM itself promote a vicious cycle that may allow for the autonomous perpetuation of the pathobiology of fibrosis $[17,18]$. Like fibrosis, tumours have been described as "wounds that do not heal" [19], and an extensive literature supports the overlapping mechanisms of wound repair and cancer. Among these mechanisms are critical roles for tumour stroma and tumour associated fibroblasts. Supporting this concept, SCHAFER and WERNER [20] reported that "the presence of large numbers of fibroblasts and myofibroblasts is a hallmark of carcinomas..." and noted that malignant cells hijack the host wound-repair response to promote formation of the tumour stroma, which is fundamental for cancer progression. Thus, the persistence of activated mesenchymal cells is critical to the pathobiology of both fibrosis and cancer.

The central role of mesenchymal cells in both cancer and fibrosis prompted us to view fibrosis through the prism of cancer biology to delineate areas in which the convergence of mechanisms might identify novel targets for intervention. Indeed, the cell phenotypes and signalling mechanisms evident in IPF and other forms of lung fibrosis recapitulate many features of malignancy. However, fibrosis is not cancer and neither the aberrant epithelial cells nor the dysfunctional mesenchymal cells of fibrotic tissue are malignant cells. Thus, we will also discuss several aspects that fundamentally distinguish the biology of cancer and fibrosis. The goal of this Back to Basics article is to discuss lung fibrosis, with an emphasis on the fibrotic lung mesenchymal cells (fibroblasts/myofibroblasts) within the context of established "hallmarks" of malignancy. In doing so, our goal is not to compare fibrotic lung cells with any specific cancer (or cancer-associated cell), but rather, to highlight the broadly overlapping cellular phenotypes and signalling paradigms that are shared by, and distinguish, cancer cells and fibrotic lung fibroblasts [21] (figure 1). We will additionally highlight selected aspects of carcinogenesis from the perspective of fibrotic disease. Because our focus is primarily on the fibrotic mesenchymal cell, the reader is referred to prior reports that discuss the overlapping biology of IPF and cancer which focus on clinical features, epithelial cell biology, genetics, epigenetics and cell-cell interactions $[2,8,11]$. "Inducing angiogenesis" is also a hallmark of cancer and the role of angiogenesis in lung fibrosis has also been reviewed recently [22]. Our hope is that ongoing comparisons between the biology of fibrosis and cancer will allow us to leverage our current understanding of each to advance innovative research hypotheses and novel therapeutic approaches for both.

\section{Fibrotic lung mesenchymal cells and the hallmarks of cancer}

Tumorigenesis represents a dynamic, multistep process leading to the malignant transformation of cells that interact with stroma and ancillary "normal" cells in a permissive microenvironment that facilitates the growth and progression of the tumour [21,23]. As a whole, tumours are complex tissues comprised of the malignant cells along with endothelial cells, immune cells, fibroblasts and stroma. Fibrotic tissues are similarly complex microenvironments comprised of abnormal epithelial cells and fibroblasts/mesenchymal cells interacting with immune cells, angiogenic pathways and ECM. In 2000 (with an update in 2011), HANAHAN and WeINBERG [21, 23] proposed a set of "hallmarks of cancer" as an organisational framework for understanding the complex biology of tumorigenesis: 1) evasion of cell death, 2) sustained proliferative signalling, 3) evading growth suppressors, 4) enabling replicative immortality, 5) activating invasion and metastasis, and 6) tumour-promoting inflammation. Here, we discuss fibrotic mesenchymal cells in the context of these hallmarks of cancer. Additionally, as tumour-promoting inflammation is considered an emerging hallmark of cancer and the role of inflammation, particularly the role of macrophages, has re-emerged as significant contributor to fibrogenesis, we include a brief discussion of macrophages in cancer and fibrosis. 


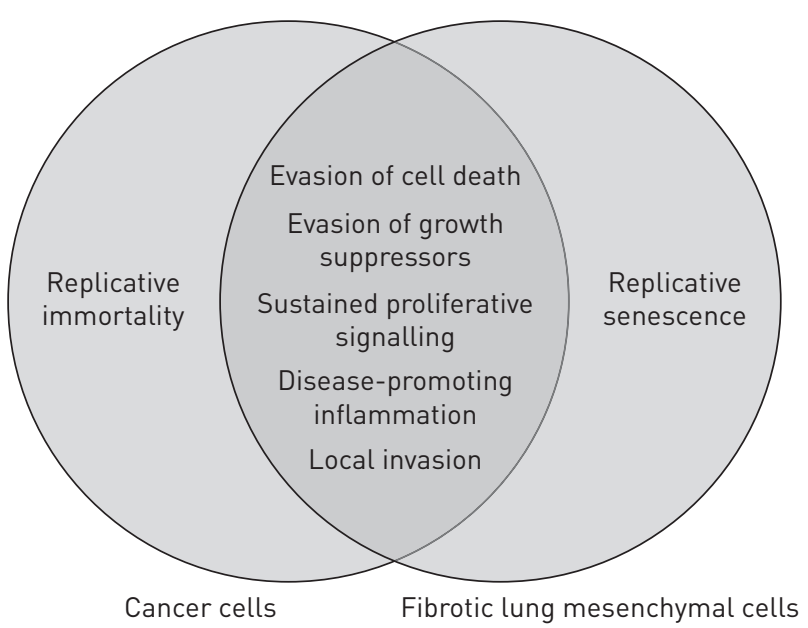

FIGURE 1 Fibrotic lung mesenchymal cells demonstrate activation of a number of signalling pathways and acquisition of cellular phenotypes that are considered to be the "hallmarks" of malignant cells and the tumour microenvironment. The replicative potential (immortality versus senescence) and capacity for invasion (potential for distant metastasis versus limitation to local invasion) represent key differences. The overlapping mechanistic biology of these processes, however, suggest that precision strategies used for therapy in cancer might be applied as antifibrotic therapies targeting the specific mesenchymal cell phenotypes that contribute to lung fibrosis.

\section{Evasion of cell death}

Cancer cells circumvent death through a variety of mechanisms that prevent the recognition, propagation and/or execution of death signals [21]. In contrast to normal wound repair, in which the majority of myofibroblasts are ultimately cleared from the microenvironment via apoptosis, fibrotic lung fibroblasts, like cancer cells, demonstrate an increased capacity to resist apoptotic stimuli [1, 24-26]. Consistently, biopsies from IPF patients show a paucity of apoptosis in the mesenchymal cells that comprise the fibroblastic foci [27-32]. As some approaches to cancer are focused on enhancing the apoptosis of malignant cells, studies of fibrotic lung fibroblasts indicate that these cells can also be sensitised to apoptotic stimuli and that lung fibrosis can be limited in vivo by targeting antiapoptotic signalling pathways [26, 33-37].

\section{Decreased responsiveness to Fas ligation}

The mechanisms responsible for mesenchymal cell resistance to apoptosis in IPF, as in cancer, are variable and perturbations affecting multiple levels of apoptosis regulation have been reported [24, 25, 34-36, 38-40]. The specific mechanisms that trigger fibroblast apoptosis during homeostatic wound repair are poorly defined. The extrinsic pathway of apoptosis, which is mediated by ligation of death receptors (such as Fas), has been implicated in the pathogenesis of lung fibrosis both through induction of epithelial cell apoptosis during the injury phase and through the capacity of fibroblasts to resist Fas-induced apoptosis during the fibrotic resolution of lung injury [40-42]. Several lines of evidence suggest that the Fas/Fas ligand (FasL) pathway is important in the regulation of lung fibroblast survival and apoptosis during lung repair and fibrosis. First, IPF lung fibroblasts express FasL, which allows them to promote ongoing injury by induction of epithelial cell apoptosis while also evading their own death by stimulating lymphocyte apoptosis [43, 44]. Second, fibrotic lung fibroblasts from IPF and murine models exhibit low expression levels of Fas, which may limit the receptor clustering necessary to initiate apoptotic signalling in response to FasL [45, 46]. Notably, Fas expression and the apoptosis susceptibility of fibroblasts can be increased by inflammatory cytokines [36]. Third, fibrotic lung fibroblasts have increased expression of c-FLIP (cellular Fas-associated protein with death domain-like interleukin-1 $\beta$-converting enzyme-inhibitory protein), which inhibits propagation of apoptotic signals downstream of Fas [40]. Fourth, fibrotic lung fibroblasts have an increased ratio of prosurvival to proapoptotic BCL-2 family proteins, favouring inhibition of the mitochondrial depolarisation that is a critical component in the apoptotic programme [34]. Finally, IPF fibroblasts have increased expression of inhibitor of apoptosis (IAP) proteins, which can prevent apoptosis via blockade of caspase activation and other effects on cell signalling $[25,34,37,39,40]$.

\section{Soluble and matrix-mediated protein kinase activation}

As with malignant cells, the apoptotic susceptibility of fibrotic lung fibroblasts can be regulated by the sustained activation of protein kinases [25, 47-51]. For example, in normal fibroblasts, the soluble profibrotic mediators transforming growth factor (TGF)- $\beta 1$ and endothelin-1 activate the prosurvival protein kinases AKT (also known as protein kinase B) and FAK (focal adhesion kinase), each of which is 
strongly linked to both lung fibrosis and cancer [47-49, 52-56]. Among their downstream signalling effectors, these protein kinases regulate expression of X-linked IAP (XIAP) and survivin, two IAP family members that are highly expressed in fibrotic lung fibroblasts and IPF tissue and have also been strongly linked with a variety of malignancies [25, 30, 39, 47, 57]. Moreover, inhibition of these IAPs promotes apoptosis in both cancer cells and fibrotic lung fibroblasts [25, 30, 37, 39, 47, 57].

Similarly, signals generated through interactions with the ECM are critical to both tumorigenesis and fibrosis. Indeed, the biochemical and biomechanical features of the fibrotic ECM have key roles in the regulation of profibrotic fibroblast phenotypes, including resistance to apoptosis [31, 50, 51, 58]. This matrix-mediated resistance to apoptosis is accomplished, in part, through suppression of protein phosphatase and tensin homologue (PTEN), a negative regulator of phosphatidylinositol 3-kinase (PI3K) and, in turn, AKT activation [31, 50, 51, 59]. Moreover, cell culture substrates that recapitulate the stiffness of fibrotic lungs are sufficient to decrease fibroblast apoptosis and increase pro-survival BCL-2 expression $[34,50,51,60]$. Increasing evidence suggests that myocardin-related transcription factor (MRTF)-A may play a key role in the integration of soluble and matrix-derived profibrotic signals. As a transcriptional co-activator that partners with serum response factor, MRTF-A regulates myofibroblast differentiation and survival [35]. MRTF-A nuclear localisation is promoted by TGF- $\beta 1$ and by increased ECM stiffness, while inhibition or deletion of MRTF impairs TGF- $\beta 1$-induced XIAP expression, increases fibroblast susceptibility to apoptosis, and attenuates lung fibrosis in murine models [34, 35, 60, 61]. Investigation of MRTF-A in the context of malignancy represents an emerging opportunity, with some studies supporting its role in tumour progression and metastasis $[62,63]$

\section{Epigenetic regulation of cell survival}

Epigenetic mechanisms can contribute to the pathogenesis of malignancy and the stability of apoptosis resistance in IPF fibroblasts through passage in cell culture supports a role for the epigenetic regulation of this phenotype in fibrosis. Such a role for epigenetic regulation was demonstrated by a study showing that a histone deacetylase inhibitor reversed the suppression of Fas/CD95 in murine fibrotic lung fibroblasts, restoring the sensitivity of these cells to apoptosis [46]. Consistent with this, another study showed that a histone deacetylase inhibitor increased the apoptosis susceptibility of IPF fibroblasts and reduced bleomycin-induced fibrosis in mice [64]. Finally, a study showed increased resistance to apoptosis in a subset of IPF fibroblasts that had decreased expression of the tumour suppressor protein $\mathrm{p} 14^{\mathrm{ARF}}$ due to increased promoter methylation of the encoding CDKN2A gene [38].

\section{Autophagy and fibroblast survival}

Autophagy is a distinct process involved in the homeostatic regulation and turnover of cytoplasmic organelles via the lysosomal pathway. It is also a process involved in the regulation of cell survival. In some circumstances, such as states of nutrient deprivation, activation of autophagy can serve as a temporary adaptive survival mechanism. In other settings, however, activation of autophagy promotes cell death (through classical apoptosis or through the overlapping mechanism of "autophagic cell death") [65]. Circumvention of death by autophagy is another mechanism implicated in the evasion of cell death during tumorigenesis [21]. Recent studies have consistently shown evidence of impaired autophagy in IPF, murine models of lung fibrosis and fibroblasts [66-72]. Specifically, TGF- $\beta 1$ activation of AKT has been shown to inhibit autophagy in lung fibroblasts [66, 68, 72]. Moreover, nintedanib, a tyrosine kinase inhibitor clinically approved for the treatment of IPF, increases autophagy in IPF fibroblasts, suggesting a novel mechanism of action for this drug [71]. In this regard, impaired autophagy may represent another mechanism by which fibrotic lung fibroblasts acquire resistance to cell death, and represents another mechanistic similarity between fibrosis and cancer $[66,68]$. Additional investigation into the regulation of autophagy and its mechanistic impact on lung fibroblast phenotypes in the context of fibrosis could shed light on these similarities and promote novel antifibrotic interventions.

\section{Sustained proliferative signalling}

Sustained proliferative signalling has been described as "arguably the most fundamental trait of cancer cells" [21]. The role of mesenchymal cell proliferation as a critical component in the maintenance and progression of fibrosis in vivo is less clear, and substantial heterogeneity has been shown in the proliferative rates of fibroblasts isolated from patients with lung fibrosis and among subpopulations of fibroblasts from individual patients [73]. Nevertheless, evidence strongly supports the persistent activation of signalling pathways that are linked to proliferation in fibrotic lung fibroblasts. Moreover, activation of these pathways contributes to the pathogenesis of lung fibrosis in murine models. The mechanisms responsible for this sustained proliferative signalling have not been clearly elucidated, although autocrine induction, liberation of sequestered growth factors and paracrine interactions with injured epithelial cells have been identified $[74,75]$. 
Activation of proliferative signalling networks that are strongly implicated in malignancy and mediated by protein kinases, such as the receptors for vascular endothelial growth factor (VEGF), platelet-derived growth factor (PDGF) and fibroblast growth factor (FGF), have also been documented in IPF fibroblasts (recently reviewed by GRIMMINGER et al. [74]). Indeed, nintedanib, a broad-spectrum tyrosine kinase inhibitor that targets the receptors for VEGF, PDGF and FGF, and was developed as an anticancer therapy due to its suppressive effects on angiogenesis, is now an approved therapy for IPF that can slow clinical progression in patients and suppress fibroblast proliferation in vitro $[5,76,77]$. Pirfenidone, which is also approved for therapeutic use for IPF, has an unknown molecular target but has also been shown to decrease fibroblast proliferation in vitro $[4,78]$.

The PI3K/AKT pathway is activated by multiple exogenous stimuli including TGF- $\beta 1$, endothelin- 1 and ECM ligation of cell-surface integrins [48, 49, 59, 79]. Increased AKT activation is observed in the fibroblastic foci of IPF patients and is associated with lung fibrosis in murine models [55, 59, 80]. Consistent with a role in the pathogenesis of fibrosis, inhibition of PI3K/AKT and its associated signalling pathways attenuates lung fibrosis in murine models [55, 81-83]. At the cellular level, in addition to its previously discussed role in apoptosis resistance, this pathway has also been implicated in lung fibroblast proliferation in vitro, as its inhibition has been shown to prevent fibroblast proliferation [2, 84, 85]. Additionally, $\beta 1$-integrin-induced PI3K/AKT activation permits fibroblasts to circumvent the antiproliferative signals transmitted to normal fibroblasts by polymerised collagen $[59,86]$.

Although studies demonstrate sustained activation of proliferative signalling pathways in IPF fibroblasts, the role of excessive fibroblast proliferation as a pathogenic mechanism of IPF is unclear, and may represent a significant difference between malignant cells and IPF fibroblasts. A number of studies conflict with the idea of excessive fibroblast proliferation in IPF and report that IPF fibroblasts actually have a decreased proliferative capacity when compared to normal fibroblasts. In one study, fibroblasts isolated from patients with active alveolitis (including both IPF and sarcoidosis) had increased proliferative rates [87]. However, the same study and others reported decreased [87-89] or unchanged [90] rates of proliferation in fibroblasts isolated specifically from IPF lung tissue. These studies also reported that IPF fibroblasts have decreased responsiveness to mitogens including serum and PDGF [87-90]. Moreover, evidence demonstrating robust cell proliferation within fibroblastic foci of IPF lung biopsies is lacking [29, 32, 91]. Collectively, these studies show that the activation of proliferative signalling pathways in IPF fibroblasts is not definitively linked with fibroblast proliferation in vivo, and that explanted fibrotic lung fibroblasts have heterogeneous baseline and mitogen-induced proliferation in vitro despite the persistent activation of these signalling pathways. Nevertheless, inhibition of signalling pathways that regulate fibroblast proliferation is associated with decreased progression of fibrosis in patients with IPF, demonstrating the need for a better understanding of the role of fibroblast proliferation in the initiation and progression of lung fibrosis.

\section{Evading growth suppressors}

Like cancer cells, fibrotic lung fibroblasts have the capacity to evade growth-suppressive signals. For example, IPF fibroblasts have diminished responsiveness to the suppressive effects of prostaglandin (PG) $E_{2}$, an eicosanoid mediator that inhibits proliferation, differentiation and collagen synthesis while enhancing susceptibility to apoptosis in normal lung fibroblasts [30, 42, 92]. As noted, IPF fibroblasts resist the growth-suppressive effects of polymerised collagen [86]. These mechanisms may be linked, as extracellular matrices that recapitulate the biomechanical stiffness of fibrotic lung tissue suppress $\mathrm{PGE}_{2}$ production by fibroblasts [51].

\section{Enabling replicative immortality}

Age is a risk factor for many cancers and for IPF but the mechanisms by which ageing contributes to the pathobiology of these processes represent a key distinction between them [21,93]. Replicative immortality is a hallmark of malignancy [21]. As noted previously, however, excessive myofibroblast proliferation is not readily evident in established lung fibrosis and, in stark contrast, accelerated replicative senescence is emerging as a critical phenotype of IPF fibroblasts $[26,94]$. Telomeres are specialised structures that protect the ends of chromosomes from deterioration, and telomerase is an enzyme that functions to lengthen telomeres and counteract the progressive telomere shortening that occurs with cell division [21, 95]. The replicative potential of normal cells is dictated by telomere length and, despite telomerase activity, continued cell division ultimately leads to senescence, which is the inability of a cell to continue to divide. Therein lies a key distinction between fibrotic lung fibroblasts and cancer cells: the sustained proliferative capacity of cancer cells requires the circumvention of cellular senescence, which can be achieved by enhanced telomerase expression and/or activity [21]. However, accelerated telomere shortening and impaired telomerase function is increasingly evident in both sporadic and familial forms of IPF [96]. Consistently, short telomere length has been associated with the worsened survival of patients with IPF [97]. 
Moreover, advanced age and short telomeres are associated with increased fibrosis and decreased fibroblast apoptosis in murine models [26, 98-101].

Few studies have specifically assessed telomere length in fibrotic lung fibroblasts and those that have are not consistent with telomere shortening as the mechanism of fibroblast senescence $[102,103]$. One study showed that telomere length was shortened in fewer than $4 \%$ of the IPF lung fibroblasts studied [102]. Interestingly, another study from the same group reported increased activity of TERT (telomerase transcriptase) in lung fibroblasts from patients with IPF and in fibroblasts from mice with experimentally induced lung fibrosis [103]. The contrast between data derived from IPF patients demonstrating short telomeres and impaired telomerase, and murine studies indicating normal telomere length and increased telomerase expression highlights an ongoing need to delineate the mechanisms linking aging and fibrosis [94].

\section{Activating invasion and metastasis}

The capacity to invade or inappropriately migrate through connective tissue planes into nonhomeostatic niches is a characteristic of malignant cells [21]. A feature of fibroblasts in pulmonary fibrosis is the ability to invade through basement membranes and ECM, and emerging studies demonstrate that IPF lung fibroblasts can be distinguished from normal lung fibroblasts by their acquisition of an invasive phenotype [104-107]. One study showed the lung fibroblasts from mice that are deficient in $\beta$-arrestin, which are protected from bleomycin-induced lung fibrosis, migrate normally but have an impaired ability to invade into Matrigel [105]. Another study showed that IPF fibroblasts have an increased ability to invade Matrigel that is dependent on expression of hyaluronan synthase (HAS) and the hyaluronan receptor CD44 [106]. Consistent with a role in fibrosis, antibody-mediated inhibition of CD44 or mesenchymal cell-specific deletion of HAS decreased lung fibrosis in a murine model [106]. Moreover, the loss of PTEN is associated with increased HAS activity and increased matrigel invasion by fibroblasts [108]. Another study showed that TGF- $\beta 1$ increases fibroblast expression of Toll-like receptor (TLR) 9 , which is linked to rapid progression of IPF. Stimulation of TLR9 significantly increased fibroblast expression of CD44 and matrix metalloproteinase-14 while promoting invasion into Matrigel [109]. Finally, a recent study indicates that while fibrotic lung fibroblasts have increased invasiveness at baseline, they also have an amplified invasive response to bronchoalveolar lavage fluid obtained from fibrotic lungs [107]. These studies demonstrate that local invasion of fibroblasts through basement membrane and interstitium can contribute to the pathogenesis of lung fibrosis, including IPF. In that regard, these cells are "cancer like". However, unlike cancers, which can acquire further invasive mechanisms that allow for distant dissemination, fibrotic lung fibroblasts demonstrate a restriction to local invasion.

\section{Tumour (and fibrosis)-promoting inflammation}

Once thought of as a gatekeeper of tumorigenesis and a bystander of progressive tumour growth and local tissue destruction, inflammation is now considered to be an enabling factor in the acquisition and persistence of the hallmark malignant phenotypes [21, 110, 111]. Innate immune cells are critical components of the tumour microenvironment that interact with both the tumour and the stromal cells. Tumour-associated macrophages (TAMs) are uniquely positioned within this microenvironment to support tumour growth, as evidenced by their ability to promote angiogenesis, activate mesenchymal cells, remodel matrix and suppress effector $\mathrm{T}$-cell responses $[112,113]$. The elucidation of these tumourimmune interactions has led to a dramatic paradigm shift in the field of immunotherapy, and led to the development of numerous novel and successful therapeutics, including a class of immune checkpoint inhibitors targeting PDL-1 (programmed cell death ligand-1), PD-1 (programmed cell death protein-1) or CTLA-4 (cytotoxic T-lymphocyte-associated protein 4) [114].

The contribution of inflammation to pulmonary fibrosis has been investigated and, at times, fiercely debated [115]. Reminiscent of the paradigm shift observed in the field of tumour immunobiology, new studies are helping us understand and appreciate how immune cells, especially monocytes and macrophages, promote fibrogenesis through molecular mechanisms other than traditional "proinflammatory" pathways. Similar to TAMs, fibrosis-associated macrophages (FAMs) display a predominant alternatively activated or M2 phenotype, best characterised by strong expression of arginase, chitinase-like molecules, resistin-like molecule $\alpha$ and CD206 (in mice) $[112,116,117]$. Additional similarities between TAMs and FAMs include the ability of FAMs to produce (or influence the production of) fibroblast growth factors (including PDGF and FGF2 [118]), profibrotic cytokines (including TGF- $\beta$ and interleukin (IL)-13 $[112,119,120]$ ) and plasminogen activator inhibitor (PAI)-1 [121] and matrix metalloproteinases [122]. Evidence that FAMs promote pulmonary fibrosis stems primarily from murine studies showing that ablation of these cells by liposomal clodronate, or deletion of C-C-motif chemokine receptor 2 or PAI-1 protect against lung fibrogenesis [120, 121, 123-126], whereas increasing the number of these cells or their monocyte precursors by IL-10 overexpression or adoptive transfer exacerbates disease [123, 127]. 
These alternatively activated macrophages express collagen (Col), suggesting that they may represent a subset of $\mathrm{CD}_{4} 5^{+} \mathrm{Col}^{+}$fibrocytes $[120,121]$, which are themselves implicated in the pathobiology of pulmonary fibrosis [127-129]. Early studies indicated that bone marrow-derived cells were recruited to murine lungs following bleomycin-induced lung injury and expressed collagen, but more recent studies demonstrate that fibrocytes do not contribute to the collagen deposition in murine models of lung fibrosis [130, 131]. Although the specific mechanisms remain to be elucidated, these findings indicate that their profibrotic effects are mediated by the paracrine regulation of effector functions in other cells [132]. Collectively, these studies suggest that macrophages (or their monocyte precursors) may represent attractive targets for the development of new classes of therapeutics for the treatment of human fibrotic lung disease while highlighting the importance of advancing our understanding of the relationship between the monocyte/macrophage and fibrocyte populations, and elucidation of the specific mechanisms by which these cells orchestrate fibrosis.

\section{Contributions of fibrotic cellular phenotypes to carcinogenesis in the lung}

In the preceding sections of this article, we have focused on viewing fibrosis through the prism of cancer biology with the goal of highlighting the ways in which fibrotic mesenchymal cells are similar to, and different than, cancer cells. However, it is increasingly evident that stromal cells and the stroma itself are critical for the development and progression of cancer. Accordingly, we will briefly discuss some of the emerging mechanisms by which different tumour-associated cell populations acquire functions that recapitulate critical profibrotic phenotypes and, in doing so, contribute to tumorigenesis in the lung.

\section{Lung cancer-associated fibroblasts}

As in lung development and fibrosis, mesenchymal cells are critical for tumorigenesis (comprehensively reviewed by KaLLURI and ZeISBERG [133]). TGF- $\beta$ signalling in fibroblasts, for instance, has been identified as a major determinant of the oncogenic potential of adjacent epithelia [17, 134]. Indeed, tumour bulk is comprised primarily of stroma, and tumour-associated fibroblasts can become activated by growth factors secreted by epithelial cells; at the same time, these tumour-associated fibroblasts produce growth factors (i.e. epidermal growth factors (EGFs), FGFs and TGFs) that contribute to the growth and dissemination of cancer cells. Additionally, tumour-associated fibroblasts regulate matrix rigidity and, thereby, tumour cell access to the vasculature, and provide the necessary scaffold for angiogenesis [135-137].

As in fibrosis, the population of tumour-associated fibroblasts is likely to be comprised of heterogeneous cells with different origins. Epithelial-mesenchymal transformation (or transition) (EMT) is a process that encompasses multiple phenotypic transitions including shape changes towards elongated and spindle-shaped cellular morphology, enhanced cytoplasmic cytoskeletal protein expression and activity, and the capacity for anchorage-independent growth, motility, migration and invasion [133, 138]. This epithelial-mesenchymal phenotypic transition has been clearly observed during the malignant transformation of respiratory epithelial cells, and this link is supported by evidence suggesting that the same oncoproteins that drive lung cancer formation and progression (i.e. mutant KRAS) are responsible for EMT [139, 140]. However, whether "true" EMT contributes to lung fibrogenesis has been a topic of considerable controversy [138, 141-144]. While some may consider EMT to be partial or incomplete in pulmonary fibrosis, abundant evidence supports the plasticity of alveolar epithelial cells that can, in the context of lung injury, acquire a number of mesenchymal-like phenotypic behaviours [143, 145-148]. This phenotypic transition is not limited to fibrotic lung injury, as Zuo et al. [149] recently showed that airway epithelial cells migrate distally in response to influenza H1N1 infection of mice, thereby acquiring fibroblast phenotypes. Unpublished observations from our laboratories (A. Marazioti and G.T. Stathopoulos) indicate that similar processes occur both after tobacco smoke-contained carcinogen exposure, and after bleomycin-induced lung injury and fibrosis (M. Spella et al., unpublished data). Cigarette smoking is a major risk factor for both lung cancer and fibrosis, and nicotine possesses multiple fibrogenic properties $[150,151]$. Thus, we speculate that nicotine-induced epithelial cell phenotypic plasticity represents an additional link between the pathobiology of fibrosis and cancer.

Mesothelial stem cells could also be a cell of fibroblast origin in IPF and of tumour-associated fibroblasts in lung cancer. To this end, fibrotic matrix deposition in IPF has been found to occur in a pleural-based manner to form the fibrotic reticulum [152]. In addition, pleural mesothelial cells have been identified to migrate into the lung parenchyma in response to fibrotic stimuli $[153,154]$. Interestingly, RinKEvich et al. [155] identified a mesothelial precursor lineage that gives rise to fibroblasts capable of clonal expansion in the lungs, kidney, liver and gut. The authors speculated that these mesothelium-derived fibroblasts may contribute to tumour stroma. Supportive of this work, unpublished observations from our laboratories indicate that lung adenocarcinomas induced by tobacco-related carcinogens overexpress, among other molecules, mesothelin (M. Spella et al., unpublished data). Hence, airway- or pleura-originated progenitors could contribute to the pool of mesenchymal cells in both IPF and lung cancer, suggesting that the mechanisms regulating the transitional plasticity of epithelial cells might be targeted for therapy in both cancer and fibrosis. 
Profibrotic signalling in lung carcinoma cells

In addition to the involvement of fibroblasts in lung cancer development and progression, fibroblast-like changes occur within lung cancer cells. First, FGF and TGF signalling in lung tumour cells provides mitogenic stimuli required for lung cancer cell proliferation and evasion of cell death [156]. Second, mutations in the various FGF receptors (FGFRs) were recently identified in non-small cell cancers of all histologic subtypes, but especially squamous cell carcinomas [157], and FGFR2 activation via perturbation of its kinase domain has been shown to be sufficient for lung adenocarcinoma development [158]. Third and most important, in addition to its aforementioned clinical impact in IPF, targeting of FGFR signalling provides significant benefits in pre-clinical models of lung cancer [156, 158]. Finally, lung tumours overexpress several fibroblast markers, which, in turn, have been found to promote tumour growth and metastasis. One of many examples is S100A4, also known as fibroblast-specific protein-1 [159]. S100A4, a proximal activator of fibroblast-specific transcriptional programmes, has been shown to be expressed in IPF [160]. At the same time, multiple studies have identified that the protein is a gatekeeper of metastatic colonisation [161, 162]. Collectively, these and other studies from the published literature strongly indicate that signalling pathways intrinsic to fibroblasts are at play in lung tumour cells, and significantly contribute to tumour cell growth and metastatic colonisation.

\section{Conclusions: precision medicine and beyond}

In cancer and in fibrosis, the precise integration and orchestration of normal wound-repair breaks down. The proximate cause(s) of the breakdown are variable and, in many cases, unknown, but the result in each case is the maintenance and propagation of aberrant cells resulting in tissue dysfunction. In addition to aberrant cell behaviour, it is increasingly evident that the matrix is not an innocent bystander to the pathological responses but is, instead, a critical contributor that is necessary for the propagation of each $[1,163,164]$.

There is substantial overlap in the phenotypes observed in cancer cells and fibrotic lung fibroblasts, yet the specific genetic and cellular mechanisms that lead to the aberrant cell behaviours observed in individuals diagnosed with cancer and fibrosis are heterogeneous [165-167]. This heterogeneity in cancer biology has spurred an increasing emphasis on developing and implementing "precision medicine" strategies that identify individual variation and employ therapeutics targeting specific mechanisms that are identified as drivers of individual cancers $[165,166]$. This precision approach has been employed successfully in the diagnostic and therapeutic approach to a number of cancers, including recent applications to some forms of lung cancer driven by mutations in the EGF receptor or the anaplastic lymphoma kinase $(A L K)$ gene $[168,169]$.

We speculate that, like cancer, heterogeneity in the inciting stimuli, genetic predisposition and signalling mechanisms that promote profibrotic cell phenotypes contribute to the variable clinical courses observed in patients with IPF. If so, then the approach to IPF, and fibrotic disease in general, may benefit from a similar precision medicine approach. Such an approach would necessitate the exploration of novel pharmacological and biological compounds, the repurposing of existing compounds, and re-examination of previously "failed" compounds in specific populations categorised by their individual genetic and cellular mechanisms. Toward that end, we propose that the shared mechanisms evident in fibrotic lung fibroblasts and cancer cells present an opportunity to leverage the wealth of existing research in cancer biology to identify novel biomarkers that reflect those underlying mechanisms and to employ those mechanistic biomarkers to stratify patients for specific interventions selected to target those mechanisms.

\section{Summary}

In summary, lung fibrosis and cancer are distinct, but share a number of cell signalling and phenotypic features. Given this mechanistic overlap combined with the substantial heterogeneity observed in both pathological processes, we speculate that strategies currently employed for the diagnosis and precision treatment of cancer might be leveraged to advance our understanding and treatment of lung fibrosis.

\section{References}

1 Thannickal VJ, Henke CA, Horowitz JC, et al. Matrix biology of idiopathic pulmonary fibrosis: a workshop report of the national heart, lung, and blood institute. Am J Pathol 2014; 184: 1643-1651.

2 Vancheri C, Failla M, Crimi N, et al. Idiopathic pulmonary fibrosis: a disease with similarities and links to cancer biology. Eur Respir J 2010; 35: 496-504.

3 Hutchinson J, Fogarty A, Hubbard R, et al. Global incidence and mortality of idiopathic pulmonary fibrosis: a systematic review. Eur Respir J 2015; 46: 795-806.

$4 \quad$ King TE Jr, Bradford WZ, Castro-Bernardini S, et al. A phase 3 trial of pirfenidone in patients with idiopathic pulmonary fibrosis. N Engl J Med 2014; 370: 2083-2092.

5 Richeldi L, du Bois RM, Raghu G, et al. Efficacy and safety of nintedanib in idiopathic pulmonary fibrosis. N Engl J Med 2014; 370: 2071-2082.

Artinian V, Kvale PA. Cancer and interstitial lung disease. Curr Opin Pulm Med 2004; 10: 425-434.

Le Jeune I, Gribbin J, West J, et al. The incidence of cancer in patients with idiopathic pulmonary fibrosis and sarcoidosis in the UK. Respir Med 2007; 101: 2534-2540. 
Antoniou KM, Tomassetti S, Tsitoura E, et al. Idiopathic pulmonary fibrosis and lung cancer: a clinical and pathogenesis update. Curr Opin Pulm Med 2015; 21: 626-633.

Raghu G, Amatto VC, Behr J, et al. Comorbidities in idiopathic pulmonary fibrosis patients: a systematic literature review. Eur Respir J 2015; 46: 1113-1130.

10 Stella GM, Inghilleri S, Pignochino Y, et al. Activation of oncogenic pathways in idiopathic pulmonary fibrosis. Transl Oncol 2014; 7: 650-655.

11 Konigshoff M. Lung cancer in pulmonary fibrosis: tales of epithelial cell plasticity. Respiration 2011; 81: 353-358

12 Lee T, Park JY, Lee HY, et al. Lung cancer in patients with idiopathic pulmonary fibrosis: clinical characteristics and impact on survival. Respir Med 2014; 108: 1549-1555.

13 Tomassetti S, Gurioli C, Ryu JH, et al. The impact of lung cancer on survival of idiopathic pulmonary fibrosis. Chest 2015; 147: 157-164.

14 Vancheri C. Common pathways in idiopathic pulmonary fibrosis and cancer. Eur Respir Rev 2013; 22: $265-272$.

15 Vancheri C. Idiopathic pulmonary fibrosis and cancer: do they really look similar? BMC Med 2015; $13: 220$.

16 Vancheri C, du Bois RM. A progression-free end-point for idiopathic pulmonary fibrosis trials: lessons from cancer. Eur Respir J 2013; 41: 262-269.

17 Horowitz JC, Thannickal VJ. Epithelial-mesenchymal interactions in pulmonary fibrosis. Semin Respir Crit Care Med 2006; 27: 600-612.

18 Blackwell TS, Tager AM, Borok Z, et al. Future directions in idiopathic pulmonary fibrosis research. An NHLBI workshop report. Am J Respir Crit Care Med 2014; 189: 214-222.

19 Dvorak HF. Tumors: wounds that do not heal. Similarities between tumor stroma generation and wound healing. N Engl J Med 1986; 315: 1650-1659.

20 Schafer M, Werner S. Cancer as an overhealing wound: an old hypothesis revisited. Nat Rev Mol Cell Biol 2008; 9: 628-638.

21 Hanahan D, Weinberg RA. Hallmarks of cancer: the next generation. Cell 2011; 144: 646-674.

22 Hanumegowda C, Farkas L, Kolb M. Angiogenesis in pulmonary fibrosis: too much or not enough? Chest 2012; 142: 200-207.

23 Hanahan D, Weinberg RA. The hallmarks of cancer. Cell 2000; 100: 57-70.

24 Thannickal VJ, Horowitz JC. Evolving concepts of apoptosis in idiopathic pulmonary fibrosis. Proc Am Thorac Soc 2006; 3: 350-356.

25 Ajayi IO, Sisson TH, Higgins PD, et al. X-linked inhibitor of apoptosis regulates lung fibroblast resistance to Fas-mediated apoptosis. Am J Respir Cell Mol Biol 2013; 49: 86-95.

26 Hecker L, Logsdon NJ, Kurundkar D, et al. Reversal of persistent fibrosis in aging by targeting Nox4-Nrf2 redox imbalance. Sci Transl Med 2014; 6: 231 ra247.

27 Korfei M, Ruppert C, Mahavadi P, et al. Epithelial endoplasmic reticulum stress and apoptosis in sporadic idiopathic pulmonary fibrosis. Am J Respir Crit Care Med 2008; 178: 838-846.

28 Kuwano K, Kunitake R, Kawasaki M, et al. P21Waf1/Cip1/Sdil and p53 expression in association with DNA strand breaks in idiopathic pulmonary fibrosis. Am J Respir Crit Care Med 1996; 154: 477-483.

29 Lepparanta O, Pulkkinen V, Koli K, et al. Transcription factor GATA-6 is expressed in quiescent myofibroblasts in idiopathic pulmonary fibrosis. Am J Respir Cell Mol Biol 2010; 42: 626-632.

30 Maher TM, Evans IC, Bottoms SE, et al. Diminished prostaglandin E2 contributes to the apoptosis paradox in idiopathic pulmonary fibrosis. Am J Respir Crit Care Med 2010; 182: 73-82.

31 Nho RS, Peterson M, Hergert $\mathrm{P}$, et al. FoxO3a (Forkhead box O3a) deficiency protects idiopathic pulmonary fibrosis (IPF) fibroblasts from type I polymerized collagen matrix-induced apoptosis via caveolin-1 (cav-1) and Fas. PloS One 2013; 8: e61017.

32 Akram KM, Lomas NJ, Forsyth NR, et al. Alveolar epithelial cells in idiopathic pulmonary fibrosis display upregulation of TRAIL, DR4 and DR5 expression with simultaneous preferential over-expression of pro-apoptotic marker p53. Int J Clin Exp Pathol 2014; 7: 552-564.

33 Ashkenazi A. Targeting the extrinsic apoptotic pathway in cancer: lessons learned and future directions. J Clin Invest 2015; 125: 487-489.

34 Zhou Y, Huang X, Hecker L, et al. Inhibition of mechanosensitive signaling in myofibroblasts ameliorates experimental pulmonary fibrosis. J Clin Invest 2013; 123: 1096-1108.

35 Sisson TH, Ajayi IO, Subbotina N, et al. Inhibition of myocardin-related transcription factor/serum response factor signaling decreases lung fibrosis and promotes mesenchymal cell apoptosis. Am J Pathol 2015; 185: 969-986.

36 Wynes MW, Edelman BL, Kostyk AG, et al. Increased cell surface Fas expression is necessary and sufficient to sensitize lung fibroblasts to Fas ligation-induced apoptosis: implications for fibroblast accumulation in idiopathic pulmonary fibrosis. J Immunol 2011; 187: 527-537.

37 Ashley SL, Sisson TH, Wheaton AK, et al. Targeting inhibitor of apoptosis proteins protects from bleomycin-induced lung fibrosis. Am J Respir Cell Mol Biol 2015 [In press DOI: 10.1165/rcmb.2015-0148OC].

38 Cisneros J, Hagood J, Checa M, et al. Hypermethylation-mediated silencing of p14 ${ }^{\mathrm{ARF}}$ in fibroblasts from idiopathic pulmonary fibrosis. Am J Physiol Lung Cell Mol Physiol 2012; 303: L295-L303.

39 Sisson TH, Maher TM, Ajayi IO, et al. Increased survivin expression contributes to apoptosis-resistance in IPF fibroblasts. Adv Biosci Biotechnol 2012; 3: 657-664.

40 Golan-Gerstl R, Wallach-Dayan SB, Zisman P, et al. Cellular FLICE-like inhibitory protein deviates myofibroblast fas-induced apoptosis toward proliferation during lung fibrosis. Am J Respir Cell Mol Biol 2012; 47: 271-279.

41 Wallach-Dayan SB, Elkayam L, Golan-Gerstl R, et al. Cutting edge: FasL ${ }^{+}$immune cells promote resolution of fibrosis. J Autoimmun 2015; 59: 67-76.

42 Huang SK, White ES, Wettlaufer SH, et al. Prostaglandin $\mathrm{E}_{2}$ induces fibroblast apoptosis by modulating multiple survival pathways. FASEB J 2009; 23: 4317-4326.

43 Wallach-Dayan SB, Golan-Gerstl R, Breuer R. Evasion of myofibroblasts from immune surveillance: a mechanism for tissue fibrosis. Proc Natl Acad Sci USA 2007; 104: 20460-20465.

44 Golan-Gerstl R, Wallach-Dayan SB, Amir G, et al. Epithelial cell apoptosis by fas ligand-positive myofibroblasts in lung fibrosis. Am J Respir Cell Mol Biol 2007; 36: 270-275.

45 Frankel SK, Cosgrove GP, Cha SI, et al. TNF- $\alpha$ sensitizes normal and fibrotic human lung fibroblasts to Fas-induced apoptosis. Am J Respir Cell Mol Biol 2006; 34: 293-304. 
Huang SK, Scruggs AM, Donaghy J, et al. Histone modifications are responsible for decreased Fas expression and apoptosis resistance in fibrotic lung fibroblasts. Cell Death Dis 2013; 4: e621.

Horowitz JC, Ajayi IO, Kulasekaran P, et al. Survivin expression induced by endothelin-1 promotes myofibroblast resistance to apoptosis. Int J Biochem Cell Biol 2012; 44: 158-169.

Horowitz JC, Rogers DS, Sharma V, et al. Combinatorial activation of FAK and AKT by transforming growth factor- $\beta 1$ confers an anoikis-resistant phenotype to myofibroblasts. Cell signal 2007; 19: 761-771.

Kulasekaran P, Scavone CA, Rogers DS, et al. Endothelin-1 and transforming growth factor- $\beta 1$ independently induce fibroblast resistance to apoptosis via AKT activation. Am J Respir Cell Mol Biol 2009; 41: 484-493.

Booth AJ, Hadley R, Cornett AM, et al. Acellular normal and fibrotic human lung matrices as a culture system for in vitro investigation. Am J Respir Crit Care Med 2012; 186: 866-876.

Liu F, Mih JD, Shea BS, et al. Feedback amplification of fibrosis through matrix stiffening and COX-2 suppression. J Cell Biol 2010; 190: 693-706.

Steelman LS, Stadelman KM, Chappell WH, et al. Akt as a therapeutic target in cancer. Expert Opin Ther Targets 2008; 12: 1139-1165.

Sulzmaier FJ, Jean C, Schlaepfer DD. FAK in cancer: mechanistic findings and clinical applications. Nat Rev Cancer 2014; 14: 598-610.

Lagares D, Kapoor M. Targeting focal adhesion kinase in fibrotic diseases. BioDrugs 2013; 27: 15-23.

Vittal R, Horowitz JC, Moore BB, et al. Modulation of prosurvival signaling in fibroblasts by a protein kinase inhibitor protects against fibrotic tissue injury. Am J Pathol 2005; 166: 367-375.

Martini M, De Santis MC, Braccini L, et al. PI3K/AKT signaling pathway and cancer: an updated review. Ann Med 2014; 46: 372-383.

Fulda S, Vucic D. Targeting IAP proteins for therapeutic intervention in cancer. Nat Rev Drug Discov 2012; 11 109-124.

Parker MW, Rossi D, Peterson M, et al. Fibrotic extracellular matrix activates a profibrotic positive feedback loop. J Clin Invest 2014; 124: 1622-1635.

Xia H, Diebold D, Nho R, et al. Pathological integrin signaling enhances proliferation of primary lung fibroblasts from patients with idiopathic pulmonary fibrosis. J Exp Med 2008; 205: 1659-1672.

Huang X, Yang N, Fiore VF, et al. Matrix stiffness-induced myofibroblast differentiation is mediated by intrinsic mechanotransduction. Am J Respir Cell Mol Biol 2012; 47: 340-348.

Bernau K, Ngam C, Torr EE, et al. Megakaryoblastic leukemia-1 is required for the development of bleomycin-induced pulmonary fibrosis. Respir Res 2015; 16: 45.

Medjkane S, Perez-Sanchez C, Gaggioli C, et al. Myocardin-related transcription factors and SRF are required for cytoskeletal dynamics and experimental metastasis. Nat Cell Biol 2009; 11: 257-268.

Scharenberg MA, Chiquet-Ehrismann R, Asparuhova MB. Megakaryoblastic leukemia protein-1 (MKL1): Increasing evidence for an involvement in cancer progression and metastasis. Int J Biochem Cell Biol 2010; 42: 1911-1914.

Sanders YY, Hagood JS, Liu H, et al. Histone deacetylase inhibition promotes fibroblast apoptosis and ameliorates pulmonary fibrosis in mice. Eur Respir J 2014; 43: 1448-1458.

Marino G, Niso-Santano M, Baehrecke EH, et al. Self-consumption: the interplay of autophagy and apoptosis. Nat Rev Mol Cell Biol 2014; 15: 81-94.

Patel AS, Lin L, Geyer A, et al. Autophagy in idiopathic pulmonary fibrosis. PloS One 2012; 7: e41394.

Ricci A, Cherubini E, Scozzi D, et al. Decreased expression of autophagic beclin 1 protein in idiopathic pulmonary fibrosis fibroblasts. J Cell Physiol 2013; 228: 1516-1524.

Nho RS, Hergert P. IPF fibroblasts are desensitized to type I collagen matrix-induced cell death by suppressing low autophagy via aberrant Akt/mTOR kinases. PloS One 2014; 9: e94616.

Gui YS, Wang L, Tian X, et al. mTOR overactivation and compromised autophagy in the pathogenesis of pulmonary fibrosis. PloS One 2015; 10: e0138625.

Im J, Hergert P, Nho RS. Reduced FoxO3a expression causes low autophagy in idiopathic pulmonary fibrosis fibroblasts on collagen matrix. Am J Physiol Lung Cell Mol Physiol 2015; 309: L552-L561.

Rangarajan S, Kurundkar A, Kurundkar D, et al. Novel mechanisms for the anti-fibrotic action of nintedanib. Am J Respir Cell Mol Biol 2016; 54: 51-59.

Sosulski ML, Gongora R, Danchuk S, et al. Deregulation of selective autophagy during aging and pulmonary fibrosis: the role of TGFß1. Aging Cell 2015; 14: 774-783.

Jordana M, Schulman J, McSharry C, et al. Heterogeneous proliferative characteristics of human adult lung fibroblast lines and clonally derived fibroblasts from control and fibrotic tissue. Am Rev Respir Dis 1988; 137: 579-584.

Grimminger F, Gunther A, Vancheri C. The role of tyrosine kinases in the pathogenesis of idiopathic pulmonary fibrosis. Eur Respir J 2015; 45: 1426-1433.

Garneau-Tsodikova S, Thannickal VJ. Protein kinase inhibitors in the treatment of pulmonary fibrosis. Curr Med Chem 2008; 15: 2632-2640.

Hostettler KE, Zhong J, Papakonstantinou E, et al. Anti-fibrotic effects of nintedanib in lung fibroblasts derived from patients with idiopathic pulmonary fibrosis. Respir Res 2014; 15: 157.

Roth GJ, Binder R, Colbatzky F, et al. Nintedanib: from discovery to the clinic. J Med Chem 2015; 58 1053-1063.

Conte E, Gili E, Fagone E, et al. Effect of pirfenidone on proliferation, TGF- $\beta$-induced myofibroblast differentiation and fibrogenic activity of primary human lung fibroblasts. Eur J Pharm Sci 2014; 58: 13-19. Manning BD, Cantley LC. AKT/PKB signaling: navigating downstream. Cell 2007; 129: 1261-1274.

Conte E, Gili E, Fruciano M, et al. PI3K p110 $\gamma$ overexpression in idiopathic pulmonary fibrosis lung tissue and fibroblast cells: in vitro effects of its inhibition. Lab Investig 2013; 93: 566-576.

Le Cras TD, Korfhagen TR, Davidson C, et al. Inhibition of PI3 K by PX-866 prevents transforming growth factor- $\alpha$-induced pulmonary fibrosis. Am J Pathol 2010; 176: 679-686.

Korfhagen TR, Le Cras TD, Davidson CR, et al. Rapamycin prevents transforming growth factor- $\alpha$-induced pulmonary fibrosis. Am J Respir Cell Mol Biol 2009; 41: 562-572.

Kang HR, Lee CG, Homer RJ, et al. Semaphorin 7A plays a critical role in TGF- $\beta 1$-induced pulmonary fibrosis. J Exp Med 2007; 204: 1083-1093. 
Conte E, Fruciano M, Fagone E, et al. Inhibition of PI3K prevents the proliferation and differentiation of human lung fibroblasts into myofibroblasts: the role of class I P110 isoforms. PloS One 2011; 6: e24663.

Lu Y, Azad N, Wang L, et al. Phosphatidylinositol-3-kinase/Akt regulates bleomycin-induced fibroblast proliferation and collagen production. Am J Respir Cell Mol Biol 2010; 42: 432-441.

Nho RS, Hergert P, Kahm J, et al. Pathological alteration of FoxO3a activity promotes idiopathic pulmonary fibrosis fibroblast proliferation on type I collagen matrix. Am J Pathol 2011; 179: 2420-2430.

Raghu G, Chen YY, Rusch V, et al. Differential proliferation of fibroblasts cultured from normal and fibrotic human lungs. Am Rev Respir Dis 1988; 138: 703-708.

Mio T, Nagai S, Kitaichi M, et al. Proliferative characteristics of fibroblast lines derived from open lung biopsy specimens of patients with IPF (UIP). Chest 1992; 102: 832-837.

Ramos C, Montano M, Garcia-Alvarez J, et al. Fibroblasts from idiopathic pulmonary fibrosis and normal lungs differ in growth rate, apoptosis, and tissue inhibitor of metalloproteinases expression. Am J Respir Cell Mol Biol 2001; 24: 591-598.

Prasad S, Hogaboam CM, Jarai G. Deficient repair response of IPF fibroblasts in a co-culture model of epithelial injury and repair. Fibrogenesis Tissue Repair 2014; 7: 7.

Cha SI, Groshong SD, Frankel SK, et al. Compartmentalized expression of c-FLIP in lung tissues of patients with idiopathic pulmonary fibrosis. Am J Respir Cell Mol Biol 2010; 42: 140-148.

Bozyk PD, Moore BB. Prostaglandin E2 and the pathogenesis of pulmonary fibrosis. Am J Respir Cell Mol Biol 2011; 45: 445-452.

Selman M, Pardo A. Revealing the pathogenic and aging-related mechanisms of the enigmatic idiopathic pulmonary fibrosis. An integral model. Am J Respir Crit Care Med 2014; 189: 1161-1172.

Thannickal VJ, Murthy M, Balch WE, et al. Blue journal conference. Aging and susceptibility to lung disease. Am J Respir Crit Care Med 2015; 191: 261-269. (TERT) mutations. PLoS One 2010; 5: e10680.

Kropski JA, Blackwell TS, Loyd JE. The genetic basis of idiopathic pulmonary fibrosis. Eur Respir J 2015; 45: 1717-1727.

Dai J, Cai H, Li H, et al. Association between telomere length and survival in patients with idiopathic pulmonary fibrosis. Respirology 2015; 20: 947-952.

Castriotta RJ, Eldadah BA, Foster WM, et al. Workshop on idiopathic pulmonary fibrosis in older adults. Chest 2010; 138: 693-703.

Naik PK, Moore BB. Viral infection and aging as cofactors for the development of pulmonary fibrosis. Expert Rev Respir Med 2010; 4: 759-771.

Sueblinvong V, Neujahr DC, Mills ST, et al. Predisposition for disrepair in the aged lung. Am J Med Sci 2012; 344: 41-51.

Povedano JM, Martinez P, Flores JM, et al. Mice with pulmonary fibrosis driven by telomere dysfunction. Cell Rep 2015; 12: 286-299.

Liu T, Ullenbruch M, Young Choi Y, et al. Telomerase and telomere length in pulmonary fibrosis. Am J Respir Cell Mol Biol 2013; 49: 260-268.

Liu T, Yu H, Ding L, et al. Conditional knockout of telomerase reverse transcriptase in mesenchymal cells impairs mouse pulmonary fibrosis. PloS One 2015; 10: e0142547.

White ES, Thannickal VJ, Carskadon SL, et al. Integrin $\alpha 4 \beta 1$ regulates migration across basement membranes by lung fibroblasts: a role for phosphatase and tensin homologue deleted on chromosome 10. Am J Respir Crit Care Med 2003; 168: 436-442.

Lovgren AK, Kovacs JJ, Xie T, et al. $\beta$-arrestin deficiency protects against pulmonary fibrosis in mice and prevents fibroblast invasion of extracellular matrix. Sci Transl Med 2011; 3: 74ra23.

Li Y, Jiang D, Liang J, et al. Severe lung fibrosis requires an invasive fibroblast phenotype regulated by hyaluronan and CD44. J Exp Med 2011; 208: 1459-1471.

Ahluwalia N, Grasberger PE, Mugo BM, et al. Fibrogenic lung injury induces non-cell-autonomous fibroblast invasion. Am J Respir Cell Mol Biol 2015 [In press DOI: 10.1165/rcmb.2015-0040OC].

Geng J, Huang X, Li Y, et al. Phosphatase and tensin homolog deleted on chromosome 10 contributes to phenotype transformation of fibroblasts in idiopathic pulmonary fibrosis via multiple pathways. Exp Biol Med 2016; 241: 157-165.

Kirillov V, Siler JT, Ramadass M, et al. Sustained activation of toll-like receptor 9 induces an invasive phenotype in lung fibroblasts: possible implications in idiopathic pulmonary fibrosis. Am J Pathol 2015; 185: $943-957$.

Grivennikov SI, Greten FR, Karin M. Immunity, inflammation, and cancer. Cell 2010; 140: 883-899.

Porta C, Larghi P, Rimoldi M, et al. Cellular and molecular pathways linking inflammation and cancer. Immunobiology 2009; 214: 761-777.

Murray PJ, Wynn TA. Protective and pathogenic functions of macrophage subsets. Nat Rev Immunology 2011; 11: 723-737.

Noy R, Pollard JW. Tumor-associated macrophages: from mechanisms to therapy. Immunity 2014; 41: 49-61.

Topalian SL, Drake CG, Pardoll DM. Immune checkpoint blockade: a common denominator approach to cancer therapy. Cancer Cell 2015; 27: 450-461.

Behr J, Kolb M, Cox G. Treating IPF - all or nothing? A PRO-CON debate. Respirology 2009; 14: 1072-1081.

Mantovani A, Biswas SK, Galdiero MR, et al. Macrophage plasticity and polarization in tissue repair and remodelling. J Pathol 2013; 229: 176-185.

Sica A, Mantovani A. Macrophage plasticity and polarization: in vivo veritas. J Clin Invest 2012; 122: $787-795$.

Barron L, Wynn TA. Fibrosis is regulated by Th2 and Th17 responses and by dynamic interactions between fibroblasts and macrophages. Am J Physiol Gastrointest Liver Physiol 2011; 300: G723-G728.

Ballinger MN, Newstead MW, Zeng X, et al. IRAK-M promotes alternative macrophage activation and fibroproliferation in bleomycin-induced lung injury. J Immunol 2015; 194: 1894-1904.

Osterholzer JJ, Olszewski MA, Murdock BJ, et al. Implicating exudate macrophages and Ly-6C high monocytes in CCR2-dependent lung fibrosis following gene-targeted alveolar injury. J Immunol 2013; 190: 3447-3457.

1 Osterholzer JJ, Christensen PJ, Lama V, et al. PAI-1 promotes the accumulation of exudate macrophages and worsens pulmonary fibrosis following type II alveolar epithelial cell injury. J Pathol 2012; 228: 170-180. 
Wynn TA. Cellular and molecular mechanisms of fibrosis. J Pathol 2008; 214: 199-210.

Gibbons MA, MacKinnon AC, Ramachandran P, et al. Ly6C $\mathrm{Ci}^{\text {hi }}$ monocytes direct alternatively activated profibrotic macrophage regulation of lung fibrosis. Am J Respir Crit Care Med 2011; 184: 569-581.

Moore BB, Paine R III, Christensen PJ, et al. Protection from pulmonary fibrosis in the absence of CCR2 signaling. J Immunol 2001; 167: 4368-4377.

Okuma T, Terasaki Y, Kaikita K, et al. C-C chemokine receptor 2 (CCR2) deficiency improves bleomycin-induced pulmonary fibrosis by attenuation of both macrophage infiltration and production of macrophage-derived matrix metalloproteinases. J Pathol 2004; 204: 594-604.

Murray LA, Chen Q, Kramer MS, et al. TGF- $\beta$ driven lung fibrosis is macrophage dependent and blocked by serum amyloid P. Int J Biochem Cell Biol 2011; 43: 154-162.

Sun L, Louie MC, Vannella KM, et al. New concepts of IL-10-induced lung fibrosis: fibrocyte recruitment and M2 activation in a CCL2/CCR2 axis. Am J Physiol Lung Cell Mol Physiol 2011; 300: L341-L353.

Andersson-Sjoland A, de Alba CG, Nihlberg K, et al. Fibrocytes are a potential source of lung fibroblasts in idiopathic pulmonary fibrosis. Int J Biochem Cell Biol 2008; 40: 2129-2140.

Mehrad B, Burdick MD, Strieter RM. Fibrocyte CXCR4 regulation as a therapeutic target in pulmonary fibrosis. Int J Biochem Cell Biol 2009; 41: 1708-1718.

Hashimoto N, Jin H, Liu T, et al. Bone marrow-derived progenitor cells in pulmonary fibrosis. J Clin Invest 2004; 113: $243-252$.

Kleaveland KR, Velikoff M, Yang J, et al. Fibrocytes are not an essential source of type I collagen during lung fibrosis. J Immunol 2014; 193: 5229-5239.

Kleaveland KR, Moore BB, Kim KK. Paracrine functions of fibrocytes to promote lung fibrosis. Expert Rev Respir Med 2014; 8: 163-172.

Kalluri R, Zeisberg M. Fibroblasts in cancer. Nat Rev Cancer 2006; 6: 392-401.

Bhowmick NA, Chytil A, Plieth D, et al. TGF- $\beta$ signaling in fibroblasts modulates the oncogenic potential of adjacent epithelia. Science 2004; 303: 848-851.

Bremnes RM, Donnem T, Al-Saad S, et al. The role of tumor stroma in cancer progression and prognosis: emphasis on carcinoma-associated fibroblasts and non-small cell lung cancer. J Thorac Oncol 2011; 6: $209-217$.

Mueller MM, Fusenig NE. Friends or foes - bipolar effects of the tumour stroma in cancer. Nat Rev Cancer 2004; 4: 839-849.

Puig M, Lugo R, Gabasa M, et al. Matrix stiffening and $\beta 1$ integrin drive subtype-specific fibroblast accumulation in lung cancer. Mol Cancer Res 2015; 13: 161-173.

Bartis D, Mise N, Mahida RY, et al. Epithelial-mesenchymal transition in lung development and disease: does it exist and is it important? Thorax 2014; 69: 760-765.

Huber MA, Azoitei N, Baumann B, et al. NF- $\kappa \mathrm{B}$ is essential for epithelial-mesenchymal transition and metastasis in a model of breast cancer progression. J Clin Invest 2004; 114: 569-581.

White RA, Neiman JM, Reddi A, et al. Epithelial stem cell mutations that promote squamous cell carcinoma metastasis. J Clin Invest 2013; 123: 4390-4404.

Chapman HA. Epithelial-mesenchymal interactions in pulmonary fibrosis. Annu Rev Physiol 2011; 73: $413-435$.

Rock JR, Barkauskas CE, Cronce MJ, et al. Multiple stromal populations contribute to pulmonary fibrosis without evidence for epithelial to mesenchymal transition. Proc Natl Acad Sci USA 2011; 108: E1475-E1483.

Duffield JS, Lupher M, Thannickal VJ, et al. Host responses in tissue repair and fibrosis. Annu Rev Pathol 2013; 8: 241-276.

Kage H, Borok Z. EMT and interstitial lung disease: a mysterious relationship. Curr Opin Pulm Med 2012; 18 517-523.

Kim KK, Wei Y, Szekeres C, et al. Epithelial cell $\alpha 3 \beta 1$ integrin links $\beta$-catenin and Smad signaling to promote myofibroblast formation and pulmonary fibrosis. J Clin Invest 2009; 119: 213-224.

Borok Z, Whitsett JA, Bitterman PB, et al. Cell plasticity in lung injury and repair: report from an NHLBI workshop, April 19-20, 2010. Proc Am Thorac Soc 2011; 8: 215-222.

Iwano M, Plieth D, Danoff TM, et al. Evidence that fibroblasts derive from epithelium during tissue fibrosis. J Clin Invest 2002; 110: 341-350.

Kim KK, Kugler MC, Wolters PJ, et al. Alveolar epithelial cell mesenchymal transition develops in vivo during pulmonary fibrosis and is regulated by the extracellular matrix. Proc Natl Acad Sci USA 2006; 103: 13180-13185.

Zuo W, Zhang T, Wu DZ, et al. $\mathrm{p} 63^{+} \mathrm{Krt}^{+}$distal airway stem cells are essential for lung regeneration. Nature 2015; 517: 616-620.

Roman J, Ritzenthaler JD, Gil-Acosta A, et al. Nicotine and fibronectin expression in lung fibroblasts: implications for tobacco-related lung tissue remodeling. Faseb J 2004; 18: 1436-1438.

2012; 26: 4778-4787.

Cool CD, Groshong SD, Rai PR, et al. Fibroblast foci are not discrete sites of lung injury or repair: the fibroblast reticulum. Am J Respir Crit Care Med 2006; 174: 654-658.

Mubarak KK, Montes-Worboys A, Regev D, et al. Parenchymal trafficking of pleural mesothelial cells in idiopathic pulmonary fibrosis. Eur Respir J 2012; 39: 133-140.

Zolak JS, Jagirdar R, Surolia R, et al. Pleural mesothelial cell differentiation and invasion in fibrogenic lung injury. Am J Pathol 2013; 182: 1239-1247.

Rinkevich Y, Mori T, Sahoo D, et al. Identification and prospective isolation of a mesothelial precursor lineage giving rise to smooth muscle cells and fibroblasts for mammalian internal organs, and their vasculature. Nat Cell Biol 2012; 14: 1251-1260.

6 Pardo OE, Latigo J, Jeffery RE, et al. The fibroblast growth factor receptor inhibitor PD173074 blocks small cell lung cancer growth in vitro and in vivo. Cancer Res 2009; 69: 8645-8651.

Cancer Genome Atlas Research Network. Comprehensive genomic characterization of squamous cell lung cancers. Nature 2012; 489: 519-525.

Tchaicha JH, Akbay EA, Altabef A, et al. Kinase domain activation of FGFR2 yields high-grade lung adenocarcinoma sensitive to a Pan-FGFR inhibitor in a mouse model of NSCLC. Cancer Res 2014; 74: 4676-4684. Venkov CD, Link AJ, Jennings JL, et al. A proximal activator of transcription in epithelial-mesenchymal transition. J Clin Invest 2007; 117: 482-491. 
160 Lawson WE, Polosukhin VV, Zoia O, et al. Characterization of fibroblast-specific protein 1 in pulmonary fibrosis. Am J Respir Crit Care Med 2005; 171: 899-907.

161 O'Connell JT, Sugimoto H, Cooke VG, et al. VEGF-A and Tenascin-C produced by S100A4 ${ }^{+}$stromal cells are important for metastatic colonization. Proc Natl Acad Sci USA 2011; 108: 16002-16007.

162 Xue C, Plieth D, Venkov C, et al. The gatekeeper effect of epithelial-mesenchymal transition regulates the frequency of breast cancer metastasis. Cancer Res 2003; 63: 3386-3394.

163 Chaudhuri O, Koshy ST, Branco da Cunha C, et al. Extracellular matrix stiffness and composition jointly regulate the induction of malignant phenotypes in mammary epithelium. Nat Mater 2014; 13: 970-978.

164 Bonnans C, Chou J, Werb Z. Remodelling the extracellular matrix in development and disease. Nat Rev Mol Cell Biol 2014; 15: 786-801.

165 Collins FS, Varmus H. A new initiative on precision medicine. N Engl J Med 2015; 372: 793-795.

166 de Bono JS, Ashworth A. Translating cancer research into targeted therapeutics. Nature 2010; 467: 543-549.

167 Obexer P, Ausserlechner MJ. X-linked inhibitor of apoptosis protein - a critical death resistance regulator and therapeutic target for personalized cancer therapy. Front Oncol 2014; 4: 197.

168 Shea M, Costa DB, Rangachari D. Management of advanced non-small cell lung cancers with known mutations or rearrangements: latest evidence and treatment approaches. Ther Adv Respir Dis 2015.

169 Schwaederle M, Zhao M, Lee JJ, et al. Impact of precision medicine in diverse cancers: a meta-analysis of phase II clinical trials. J Clin Oncol 2015; 33: 3817-3825. 